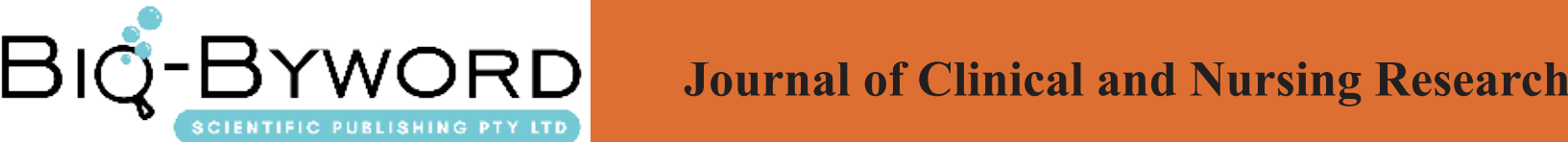

\section{Effect of Danshen Polyphenate Combined with Pelectin on Diabetic Pe- ripheral Neuropathy and Its Effects on Serum IGF-1, FFA and MBP}

\author{
Pang Jing Xu Jie Bai Xiaogang* \\ Affiliated Hospital of Yan'an University, Yan'an, Shaanxi 716000
}

\section{ARTICLE INFO}

Article history:

Published online: 15th July, 2017

\section{Key words:}

Salvia miltiorrhiza polyphenolic acid puerarin sodium diabetic peripheral neuropathy

*Corresponding Authors

\section{ABSTRACT}

Objective: To observe the therapeutic effect of salvia miltiorrhiza phenylephrine combined with puerarin sodium on diabetic peripheral neuropathy (DPN) and the effect of serum insulin-like growth factor 1 (IGF-1), free fatty acid (FFA) and human myelin Protein (MBP). Methods: A total of 116 patients with diabetic peripheral neuropathy were randomly divided into observation group (58 cases) and control group (58 cases). The control group was treated with sodium prostaglandin sodium. The observation group was treated with Danshen phenolic acid Treatment, treatment for 8 weeks. The changes of total symptom score system (TSS), vibration sensory threshold (VPT), ankle brachial index (ABI) and peripheral nerve conduction velocity (NCV), serum IGF-1, FFA and MBP were observed before and after treatment. Results: The levels of numbness, tingling, burning, paresthesia and total score were significantly decreased in both groups after treatment, VPT, $\mathrm{ABI}$ and NCV were improved $(\mathrm{P}<0.05)$, and the above indexes $(\mathrm{P}<0.05)$. The levels of serum IGF-1 were increased and the levels of FFA and MBP were lower in the two groups. The improvement of the above indexes was better than that of the control group $(\mathrm{P}<0.05)$. Conclusion: Salvia miltiorrhiza polyphenate combined with puerarin sodium can significantly improve the vibration sensory threshold and ankle-brachial index, enhance the nerve conduction velocity and relieve clinical symptoms in patients with diabetic peripheral neuropathy. The mechanism may be related to the regulation of serum IGF-1, FFA, The level of MBP is relevant. 


\section{Introduction}

Diabetic peripheral neuropathy (DPN) is the most common chronic complication in diabetic patients. Related epidemiological data show that $30 \%$ to $70 \%$ of diabetic patients may be associated with DPN, with varying degrees of limb numbness, paresthesia Burning sensation and pain and other symptoms, severe cases can occur lower extremity ulcer gangrene, and even amputation, seriously affecting the patient's physical health and quality of life ${ }^{[1]}$. At present, the pathogenesis of DPN is not completely clear, and the body metabolic disorders, oxidative stress, microvascular injury and inflammatory response, and prognosis and its therapeutic effect is closely related ${ }^{[2,3]}$. The current clinical disease for the main use of antioxidants, neurotrophic agents, vasodilators and other treatment, but the effect is not satisfied. In recent years, Chinese and Western medicine treatment program has been gradually applied in clinical, and achieved unique clinical efficacy ${ }^{[4]}$. (VPT), ankle brachial index (ABI) and peripheral nerve conduction velocity (NCV) and serum insulin-like growth factor 1 (IGF-1) in patients with DPN were significantly higher than those in control group $(\mathrm{P}<0.05)$, free fatty acids (FFA) and human myelin basic protein (MBP), the results are reported below.

\section{Clinical data}

1.1 General information. From December 2014 to April 2016 in our hospital treated 116 cases of DPN, aged 1870 years old, are in line with the 1999 World Health Organization (WHO) on the diagnostic criteria for diabetes, and in line with the Chinese Medical Association of endocrine and metabolic Medical Association in 2009 developed DPN diagnostic criteria ${ }^{[5]}$. Patients and their families signed the informed consent form, and voluntary participation in this study. Exclusion of diabetic acute complications (diabetic ketoacidosis, etc.); combined with trauma, poisoning, immunization, lumbar disease and other factors caused by peripheral neuropathy; coagulation dysfunction, severe liver and kidney dysfunction, acute cardiovascular and cerebrovascular diseases, malignant cancer, autoimmune diseases, hematopoietic system diseases, pregnancy or lactation women, cognitive dysfunction, mental disorders or other neuropsychiatric diseases; treatment compliance is poor and cannot cooperate with the examination. 116 patients with DPN were randomly divided into two groups: the observation group 58 cases, male 34 years old, female 24 cases; age $43 \sim 69(57.5 \pm 8.5)$ years; diabetes course of $5 \sim 18(10.2 \pm 2.2)$ years; fasting blood glucose $10.2 \pm 2.0) \mathrm{mmol} / \mathrm{L}$, glycosylated hemoglo- bin $(9.1 \pm 1.1) \%$. $(10.8 \pm 2.6)$ years; the fasting blood glucose $(10.8 \pm 1.5) \mathrm{mmol} / \mathrm{L}$, glycosylated hemoglobin $(10.8 \pm 1.5) \mathrm{mmol} / \mathrm{L}$, the age of $42 \sim 70(58.1 \pm$ 7.3), diabetic course of 6 to $19(10.8 \pm 2.6)$ years; fasting blood glucose $(10.8 \pm 1.5) \mathrm{mmol} / \mathrm{L}$, glycosylated hemoglobin $(9.5 \pm 1.0) \%$. (This is our request, please note that) two groups of age, gender, diabetes duration, fasting blood glucose, glycosylated hemoglobin was no significant difference (all>0.05), comparable.

1.2 Treatment. Both groups were given routine treatment of Western medicine, including Zhu Huanzhe quit smoking, weight control, control of blood sugar, nutritional nerve and improve microcirculation therapy. The control group on the basis of the above treatment to give sodium prostaglandin sodium $40 \mu \mathrm{g}$ / oral, 3 times / d; observation group in the control group based on the administration of Danshen Polyphenols 200mg by adding $5 \%$ glucose injection or $0.9 \%$ sodium chloride injection $250 \mathrm{~mL}$ in the intravenous infusion, 1 times / d. 2 weeks of treatment were 8 weeks. (This is our request, please note)

1.3 Observation indicators (1) Observed before and after treatment of the two groups before the total symptom score system (TSS) score ${ }^{[6]}$, the scoring system, including numbness, tingling, burning sensation, sensory abnormalities 4 symptoms and signs, according to the severity of no, light, medium and heavy, respectively, corresponding to $0-3$ points, and finally calculate the TSS total points. (2) The changes of VPT, ABI and NCV were observed before and after treatment, and VPT was detected by Sensioete A200 digital vibration sensory threshold check. NCV included motor conduction velocity (MNCV) and sensory conduction velocity (SNCV) of central nerve and peroneal nerve; $\mathrm{ABI}$ includes left and right ankle brachial index. (3) The levels of serum IGF-1, FFA and MBP before and after treatment were observed by immunoassay (ELISA).

1.4 Statistical methods. Using SPSS17.0 statistical software for analysis. The measurement data is expressed as $\overline{\mathrm{x}} \pm \mathrm{s}$, and the group $\mathrm{t}$ test is used. The counting data are expressed in terms of rate or composition ratio. The data processing is $\chi^{2}$ test. $\mathrm{P}<0.05$ for the difference was statistically significant.

\section{Results}

2.1 TSS scores were significantly lower in both groups before and after treatment $(\mathrm{P}<0.05)$. The scores of numbness, tingling, burning, paresthesia and total scores were significantly lower. And the above points in the observation group were significantly lower than 
Table 1 Before and after treatment TSS score comparison $(\overline{\mathrm{x}} \pm \mathrm{s}$, points $)$

\begin{tabular}{|c|c|c|c|c|c|c|c|c|c|c|}
\hline \multirow[t]{2}{*}{ Group } & \multicolumn{2}{|c|}{ Numbness } & \multicolumn{2}{|c|}{ Tingling } & \multicolumn{2}{|c|}{ Burning } & \multicolumn{2}{|c|}{ Sensation abnormal } & \multicolumn{2}{|c|}{ Total score } \\
\hline & Before & After & Before & After & Before & After & Before & After & Before & After \\
\hline $\begin{array}{l}\text { Observation } \\
\text { group }\end{array}$ & $2.73 \pm 0.22$ & $0.82 \pm 0.18^{(1)}$ & $2.51 \pm 0.31$ & $0.76 \pm 0.21^{(1)}$ & $2.38 \pm 0.50$ & $1.12 \pm 0.32^{(1)}$ & $2.81 \pm 0.10$ & $1.21 \pm 0.35^{(1)}$ & $10.97 \pm 1.19$ & $4.13 \pm 1.21^{(1)}$ \\
\hline Control group & $2.75 \pm 0.27$ & $1.16 \pm 0.23^{(1)}$ & $2.58 \pm 0.36$ & $1.05 \pm 0.30^{(1)}$ & $2.41 \pm 0.42$ & $1.47 \pm 0.49^{(1)}$ & $2.83 \pm 0.12$ & $1.58 \pm 0.31^{(1)}$ & $11.027 \pm 1.24$ & $6.27 \pm 1.42^{(1)}$ \\
\hline$t$ & 0.384 & 6.893 & 0.781 & 5.178 & 0.156 & 6.984 & 0.320 & 5.743 & 0.109 & 5.378 \\
\hline$P$ & $>0.05$ & $<0.05$ & $>0.05$ & $<0.05$ & $>0.05$ & $<0.05$ & $>0.05$ & $<0.05$ & $>0.05$ & $<0.05$ \\
\hline
\end{tabular}

Note: (1) before treatment compared to $\mathrm{P}<0.05$,

Table 2 Comparison of VPT before and after treatment $(\overline{\mathrm{x}} \pm \mathrm{s})$

\begin{tabular}{|c|l|c|c|c|c|}
\hline \multirow{2}{*}{ Group } & \multirow{2}{*}{ Cases } & \multicolumn{3}{|c|}{ Left lower limb } & \multicolumn{2}{c|}{ Right lower limb } \\
\cline { 3 - 6 } & & Before & \multicolumn{2}{|c|}{ After } & After \\
\hline $\begin{array}{c}\text { Observation } \\
\text { group }\end{array}$ & 58 & $19.27 \pm 1.50$ & $13.10 \pm 0.95^{(1)}$ & $20.29 \pm 1.78$ & $14.11 \pm 1.03^{(1)}$ \\
\hline Control group & 58 & $19.20 \pm 1.61$ & $15.84 \pm 0.89^{(1)}$ & $20.45 \pm 1.84$ & $16.51 \pm 1.22^{(1)}$ \\
\hline$t$ & & 0.583 & 4.175 & 0.174 & 4.056 \\
\hline$P$ & & $>0.05$ & $<0.05$ & $>0.05$ & $<0.05$ \\
\hline
\end{tabular}

Note: (1) before treatment compared to $\mathrm{P}<0.05$,

those in the control group $(\mathrm{P}<0.05)$. See Table 1.

2.2 VPT, ABI and NCV were compared between the two groups before and after treatment. The improvement of VPT, ABI and NCV were improved in the two groups. The improvement of the above indexes was better than that of the control group $(\mathrm{P}<0.05)$. See Tables 2,3 and 4 .

The levels of serum IGF-1, FFA and MBP in the two groups were significantly higher than those in the control group $(\mathrm{P}<0.05)$, while the levels of FFA and MBP were decreased $(\mathrm{P}<0.05)$ The improvement was better than the control group $(\mathrm{P}<0.05)$. See Table 5 .

\section{Discussion}

DPN patients due to their long-term abnormal glucose metabolism, polyol bypass activation and the deposition of glycosylated products such as peripheral nerve ede-

Table 3 Comparison of NCV before and after treatment in both groups $(\overline{\mathrm{x}} \pm \mathrm{s})$

\begin{tabular}{|c|c|c|c|c|c|}
\hline \multirow[t]{2}{*}{ Group } & \multirow[t]{2}{*}{ Cases } & \multicolumn{2}{|c|}{ MNCV } & \multicolumn{2}{|c|}{ SNCV } \\
\hline & & $\begin{array}{l}\mathrm{C} \text { e } \mathrm{n} \mathrm{t} \mathrm{r} \text { a I } \\
\text { nerve }\end{array}$ & $\begin{array}{l}\text { Peroneal } \\
\text { nerve }\end{array}$ & $\begin{array}{l}\mathrm{C} \text { e } \mathrm{n} \mathrm{t} \mathrm{r} \text { a l } \\
\text { nerve }\end{array}$ & $\begin{array}{l}\text { Peroneal } \\
\text { nerve }\end{array}$ \\
\hline \multirow[t]{4}{*}{$\begin{array}{l}\text { Observation } \\
\text { group }\end{array}$} & Before & $43.27 \pm 2.40$ & $41.17 \pm 2.50$ & $39.29 \pm 2.78$ & $42.55 \pm 2.50$ \\
\hline & After & $49.17 \pm 2.50^{1}$ & $52.71 \pm 3.20^{1}$ & $48.42 \pm 3.71^{1}$ & $50.38 \pm 2.92^{(1)}$ \\
\hline & $T$ & 5.894 & 6.721 & 5.390 & 5.094 \\
\hline & $P$ & $<0.05$ & $<0.05$ & $<0.05$ & $<0.05$ \\
\hline Control group & Before & $42.20 \pm 2.61$ & $41.22 \pm 2.61$ & $38.10 \pm 2.40$ & $42.27 \pm 2.48$ \\
\hline
\end{tabular}

Note: (1) control group compared $\mathrm{P}<0.05$, 
Table 4 Comparison of ABI before and after treatment $(\overline{\mathrm{x}} \pm \mathrm{s})$

\begin{tabular}{|c|l|c|c|c|c|}
\hline \multirow{2}{*}{ Group } & \multirow{2}{*}{ Control } & \multicolumn{3}{|c|}{ Right ankle brachial index } & \multicolumn{2}{c|}{ Left ankle brachial index } \\
\cline { 3 - 6 } & & \multicolumn{3}{|c|}{ After } & \multicolumn{3}{|c|}{ Before } \\
\hline $\begin{array}{c}\text { Observation } \\
\text { group }\end{array}$ & 58 & $0.72 \pm 0.17$ & $1.11 \pm 0.20^{1}$ & $0.75 \pm 0.14$ & $1.16 \pm 0.26^{(1)}$ \\
\hline Control group & 58 & $0.70 \pm 0.16$ & $0.98 \pm 0.19^{(1)}$ & $0.74 \pm 0.13$ & $1.02 \pm 0.24^{(1)}$ \\
\hline$T$ & & 0.105 & 8.164 & 0.321 & 5.843 \\
\hline$P$ & & $>0.05$ & $<0.05$ & $>0.05$ & $<0.05$ \\
\hline
\end{tabular}

Note: (1) before treatment compared to $\mathrm{P}<0.05$,

Table 5 Comparison of serum IGF-1, FFA and MBP levels before and after treatment $(\overline{\mathrm{x}} \pm \mathrm{s})$

\begin{tabular}{|c|c|c|c|c|c|c|c|}
\hline \multirow{2}{*}{ Group } & Cas- & \multicolumn{2}{|c}{ IGF-1(ng/L) } & \multicolumn{2}{c|}{ MBP $(\mu \mathrm{g} / \mathrm{L})$} & \multicolumn{2}{c|}{ FFA( $\mu \mathrm{mol} / \mathrm{L})$} \\
\cline { 3 - 8 } & es & Before & \multicolumn{2}{|c|}{ After } & Before & After & Before \\
\hline $\begin{array}{c}\text { Observation } \\
\text { group }\end{array}$ & 58 & $84.59 \pm 8.73$ & $163.29 \pm 12.71^{(1)}$ & $5.83 \pm 0.89$ & $1.67 \pm 0.37^{(1)}$ & $710.20 \pm 25.27$ & $412.75 \pm 20.29^{(1)}$ \\
\hline Control group & 58 & $83.87 \pm 6.49$ & $136.30 \pm 10.50^{(1)}$ & $5.80 \pm 0.92$ & $3.10 \pm 0.39^{(1)}$ & $714.17 \pm 27.44$ & $485.41 \pm 21.30^{(1)}$ \\
\hline$t$ & & 0.451 & 6.174 & 0.105 & 10.174 & 0.518 & 5.589 \\
\hline$P$ & & $>0.05$ & $<0.05$ & $>0.05$ & $<0.05$ & $>0.05$ & $<0.05$ \\
\hline
\end{tabular}

Note: (1) before treatment compared to $\mathrm{P}<0.05$,

ma necrosis, can affect the peripheral nerve conduction velocity, by electrophysiological examination can show that patients with median nerve, the motor conduction velocity and sensory conduction velocity are significantly reduced. In addition, ABN patients with DPN significantly reduced, and with the extent of peripheral neuropathy was negatively correlated, after treatment intervention, the value can be significantly increased ${ }^{[7]}$. VPT is widely used in assessing the condition of DPN patients and can be used as an important indicator of early screening for diabetic peripheral neuropathy and assessing the risk of death in patients ${ }^{[8]}$. In addition, the abnormal expression of IGF-1, FFA and MBP in the body plays an important role in the development and progression of DPN. IGF-1 is a neurotrophic factor with insulin-like function, which promotes neuronal growth and repair function. IGF-1 expression can decrease the peripheral nerve conduction velocity significantly ${ }^{[9]}$. MBP is a kind of nerve myelin contains lipoprotein, is also an important nutrient myelin nutrition, to maintain the central nervous nerve sheath structure and function of stability is of great significance, and DPN patients due to nerve fiber damage, the body can be MBP compensatory increase, therefore, increased levels of MBP to a certain extent, reflects the scope and severity of peripheral nerve damage ${ }^{[10]}$. FFA is an inflammatory and lipid metabolic disorders of inflammatory products, due to the presence of diabetes in patients with abnormal glucose and lipid metabolism, FFA can show a certain degree of increase, not only cause insulin resistance, but also lead to oxygen free radicals, thus induced DPN occurred ${ }^{[2]}$.

Betacephalron sodium is a prostacyclin analogue, which can dilate blood vessels, inhibit platelet adhesion agglutination, enhance tissue oxygen supply, inhibition of diabetes in patients with abnormal glucose and lipid metabolism caused by peripheral vascular sclerosis and stenosis, improve peripheral nerve blood supply; can inhibit adenylate cyclase, causing elevated levels of intracellular cyclic adenosine monophosphate, thereby inhibiting the migration or proliferation of vascular smooth muscle cells; also inhibit calcium influx and thromboxane A2 synthesis, reduce the risk of thrombosis, improve micro cycle. Previous reports have shown that the use of sodium metoprost sodium in the treatment of DPN can significantly improve the patient's median nerve, peroneal nerve conduction velocity and sensory conduction velocity, reduce the clinical symptoms ${ }^{[11]}$. But the simple application of the drug treatment of some patients still cannot achieve satisfactory results. 
Chinese medicine and no DPN this exclusive disease name, according to the clinical symptoms of the disease will be attributed to "pain syndrome" "Bi Syndrome" "Diabetes" and other areas. Many Chinese medicine practitioners believe that patients due to diarrhea for a long time, causing Qi Yin loss and pulse stasis, blood stagnation, leading to yang cannot reach the limbs and the incidence of disease, and that the disease is the key link for "blood stasis "[12]. Danshen polyphenols have blood circulation, sedative analgesia and other effects, which can regulate glycolipid metabolism, dilation of blood vessels, increase local tissue blood flow, anti-inflammatory and anti-oxidation, inhibition of platelet activation, protection of vascular endothelial cell function, improve microcirculation, Reduce inflammatory edema, etc. ${ }^{[13]}$. Zhou Fuqiang et al ${ }^{[14]}$ reported that Salvia polyphenolic acid in the promotion of peripheral nerve entrapment after rehabilitation in patients with a good effect, can significantly improve the edema of peripheral nerve edema, reduce capillary permeability, reduce Inflammatory mediator stimulation, is conducive to the recovery of neurological function in patients. Animal experiments confirmed that Salvia miltiorrhiza polyphenate can promote ischemia-reperfusion injury in rat brain cells in the opening of the potassium channel, reduce calcium overload, protect cell membrane integrity, inhibit endothelial cell involvement in inflammatory response, and regulate the brain tissue Brain-derived neurotrophic factor (BDNF) and glial derived neurotrophic factor (GDNF) expression, to improve neurological function to help ${ }^{[15]}$.

The results of this study showed that the levels of numbness, tingling, burning, paresthesia and total score were significantly decreased in both groups after treatment, VPT, ABI and NCV were improved, serum IGF-1 level increased, and FFA And MBP levels were lower, and the improvement of the above indicators in the observation group were better than the control group. It is suggested that Salvia Miltiorrhiza Gypsophila combined with puerarin sodium can significantly improve the vibration sensory threshold and ankle brachial index, enhance nerve conduction velocity and relieve clinical symptoms in DPN patients. The mechanism may be related to the regulation of serum FFA, MBP and IGF-1 levels.

\section{References}

[1] Wang Xiaomei. Effect of Shenqi Jiangtang Granule Combined with Ginkgo Biloba Capsule on 52 Cases of Diabetic Peripheral Neuropathy [J]. Chinese Journal of Experimental Prescription, 2015, 21 (5): 201-205

[2] Shi Jun, Zhang Wenchuan. Advances in Pathogen- esis of Diabetic Peripheral Neuropathy [J]. Journal of Shanghai Jiaotong University: Medical Science, 2012, 32 (1): 116-119

[3] Clinical Study on the Effects of TCM Internal and External Treatment on Serum IGF-1 and TNF- $\alpha$ in Patients with Diabetic Peripheral Neuropathy [J]; Journal of Chengdu University of Traditional Chinese Medicine; 2013.

[4] Lu Shuquan, Zhang Shufang, Su Xiuhai, et al. Advances In Treatment of Diabetic Peripheral Neuropathy with Traditional Chinese Medicine [J]. Journal of Liaoning University of Traditional Chinese Medicine, 2013, 15 (2): 205-207

[5] Wei Guirong. Effect of Alprostadil on Epilepsy with Diabetic Peripheral Neuropathy [J]. Chinese Journal of Gerontology, 2012, 32 (9): 1804-1805

[6] Ji Ping, Wang Shumin, Jiang Jiaming, et al. Effect of Huangqi Guizhi Wuwu Decoction on Diabetic Peripheral Neuropathy [J]. China Medical Engineering, 2014, 22 (4): $75-76$

[7] Chen Jianjun. Type 2 Diabetic Peripheral Neuropathy and Ankle Brachial Index of the Relationship [D]. Central South University, 2007

[8] Hu Xuejian, Ren Jiangong, Luo Hui, Yang Yan. Ibisatide Combined With Antioxidants In The Treatment Of Diabetic Peripheral Neuropathy In Patients With Vibration Sensory Threshold Comparison Analysis [J]. Chinese Journal of Hospital Pharmacy, 2016, 36 (12): $1022-1024$

[9] Yang Qingqing. Transforming Growth Factor B1 and Type 2 Diabetic Peripheral Neuropathy Relationship [D]. Suzhou University, 2013.

[10] Cong Yan, Li Jinxuan, Qin Xiaofeng. Serum S100 $\beta$, MBP Content And Diabetic Peripheral Neuropathy Related To The Study [J]. World Clinical Medicine, 2013, 34 (7): 409-411

[11] Liu Shiming, Zhang Juan, Cheng Baiduo. Methylcobalamin, Puerarin Sodium Oral Treatment of Diabetic Peripheral Neuropathy [J]. Modern Journal of Integrated Traditional and Western Medicine, 2015, 24 (32): 3575-3577

[12] Zhu Zhenling, Jin Zhisheng, Wei Yujiao. Chinese and Western Medicine Treatment of Diabetic Peripheral Neuropathy Research Progress [J]. Journal of Gansu College of Traditional Chinese Medicine, 2014, 31 (2): 87-90 
[13] Zhang Xiaolei, Chen Junhua, Guo Chunxia, Xin Shuliang. Danshen Polyphenolic Acid Pharmacological Effects [J]. World Clinical Medicine, 2013, 34 (5): 292 297

[14] Zhou Fuqiang, Song Peng. Salvia Miltiorrhiza Polyphenolic Acid in the Peripheral Nerve Capsule after the Release of the Efficacy of Rehabilitation [J].
Health (Academic Edition), 2014, 8 (12): 159-160

[15] Wang Qiang, Zhang Yi, Wang Rong, Yang Yilin. Effects of Salvia Miltiorrhiza Phenate on BDNF and GDNF in Brain Tissue of Rats with Ischemia Reperfusion Injury [J]. Chinese Journal of Cerebrovascular Diseases, 2010, 20 (5): 272-274 